\title{
Consumer expectations, liking and willingness to pay for specialty foods: Do sensory characteristics tell the whole story?
}

\author{
Gianluca Stefani ${ }^{\mathrm{a}, \mathrm{b}, *}$, Donato Romano ${ }^{\mathrm{a}}$, Alessio Cavicchi ${ }^{\mathrm{a}}$ \\ a Department of Agricultural and Resource Economics, University of Florence, P.le delle Cascine 18, 50144 Firenze, Italy \\ ${ }^{\mathrm{b}}$ Environment Department, The University of York, Helsington, York YO10 5DD, UK
}

Available online 24 August 2005

\begin{abstract}
The Region of origin of food products affects consumer valuation in two different ways. First, origin can act as a quality cue hinting to other characteristics of the good. Secondly, origin can affect directly the value of food due to its symbolic or affective role. This study was carried out in order to investigate the direct effect of geographical origin when the size of the area of origin shrinks and its definition becomes more precise. A valuation experiment was designed to assess the impact of origin on consumer evaluation and to analyse how it relates to WTP and hedonic scores. A well-known specialty food-spelt - that originates from three concentric areas - Garfagnana (a small valley of the Apennines), Tuscany and Italy — was chosen as a case study. Both hedonic and monetary evaluations were elicited from 77 subjects after blind tasting condition, looking at labels only and finally tasting a labelled product. Results reveal that, in the case of spelt, the narrower and more precisely defined the area of origin the higher the quality expectation of consumers supporting the role of origin as a quality cue. A direct impact of origin on willingness to pay was also found.
\end{abstract}

(C) 2005 Elsevier Ltd. All rights reserved.

Keywords: Region of origin; Hedonic scores; WTP; Experimental auctions; Italy

\section{Introduction}

The Region of origin of food products can affect consumer valuation in two different ways. First, origin acts as a quality cue hinting to other characteristics of the good such as sensory characteristics. Secondly, origin impacts directly on the value of food due to its symbolic or affective role. The general objective of this study is the analysis of the direct and overall effect of geographical origin on consumer valuation.

The paper reports results of a valuation experiment designed to provide both hedonic scores and consumers willingness to pay (WTP) under different testing

\footnotetext{
${ }^{*}$ Corresponding author. Address: Department of Agriculture and Resource Economics, University of Florence, P.le delle Cascine 18, 50144 Firenze, Italy. Tel.: +39055 3288223; fax: +39055 361771.

E-mail address: gstefani@unifi.it (G. Stefani).
}

conditions related to the origin of the product and its sensory characteristics. Alternative definitions (wider vs narrower) of the area of origin and their impacts on valuations were also explored.

Spelt was selected as a case study because it is a natural candidate for a research such as this. Spelt is a type of grain, an ancestor of wheat, that is used to prepare supper and salads in the same fashion of rice. A long tradition in spelt growing and in its culinary use is retained by Garfagnana, a small mountainous area of Tuscany. Indeed, spelt coming from Garfagnana obtained the European Protected Geographic Indication (PGI) in 1996 and can be considered a specialty food with a strong regional identity. Nevertheless, spelt from Tuscany or from Italy is also appreciated although to different degrees.

The remainder of this paper is structured as follows. In the next section the literature on the effect of 
geographical origin on consumers' attitude and valuations is reviewed and research objectives are illustrated. The methodology is explained in Section 3, while Section 4 discusses research results. Conclusions are drawn in the last section.

\section{Geographical origin and consumer choice}

Growing attention has been paid in the marketing literature to the issue of country or region of origin of foodstuffs. Several works tend to underline the cognitive role of the region of origin as a quality cue. In particular, regional products are more successful when their relevant characteristics match the consumer's image of the region in terms of natural environment or human factors depending on the raw vs transformed nature of the food (Van Ittersum, Candel, \& Meulemberg, 2003).

According to this view, the region of origin has an indirect impact on consumer preferences as a quality cue, that is a product characteristic that consumers can assess before consumption and is perceived as related to other product attributes (Verlegh \& Van Ittersum, 2001). Two types of cues can be identified: extrinsic and intrinsic cues. Intrinsic cues refer to characteristics such as physical features of the product (for instance its shape, colour and size) whilst extrinsic ones, although related to the product, are not part of its physical description. ${ }^{1}$ Assessing quality through quality cues leads to what has been named "expected" quality, whilst "experience" or "perceived" quality can be assessed only through actual consumption (Steenkamp, 1990; Grunert, Larsen, Madsen, \& Baadsgaard, 1996).

Other studies stress how geographical origin plays other more direct roles in determining consumer behaviour as it can be valued per se, that is for its symbolic and affective role, or because of the ethical and normative consequences linked to purchase or consumption (Verlegh \& Steenkamp, 1999).

As far as regional specialties are concerned, the affective dimensions relate to the sense of identity and belonging to the region of production or to the evocation of past experiences and emotions such as those experienced in occasion of holidays spent in the area (Van der Lans, Van Ittersum, De Cicco, \& Loseby, 2001). Another affective aspect refers to the sense of tradition and authenticity conveyed by any reference to the region of origin of food (Bell \& Valentine, 1997, p. 175).

Even ethical or normative issues have been related to particular regions of origins of foodstuff. The norm "buy national" that has been associated with the trait

\footnotetext{
${ }^{1}$ The marketing literature list as extrinsic cues the following characteristics among others: price, brand, region of origin, retail brand (Steenkamp, 1990).
}

of ethnocentrism at the country level may be present at the regional level too, even if its relevance may be reduced by the small share of local consumers in markets of most specialty foods. Of more relevance is the occurrence of "buycotting" behaviour (Friedman, 1996) to help producers in marginal rural areas or to sustain traditional and nearly extinct productions.

Noticeably, either for its role as quality cue or for its direct impact it can be assumed that, at least for certain food specialities, the more precisely the area of origin is defined the higher the value of the product for the consumers. As long as the success of a product depends on natural environments or human factors (Van Ittersum et al., 2003), their peculiarity and homogeneity are likely to be diluted as the definition of the region is widened. The same holds when consumers are driven by affective factors such as the search for "culinary heritage" (Bessière, 1998) or when they behave like "authenticity seekers" (Ray, 1998).

Similar assumptions can be found in the narrative about institutionalised systems of quality certification such as the one embodied by the appellation dorigine and by the European Community regulation for the wine industry (Moran, 1993). According to these schemas, the definition of the region of origin is structured in concentric areas with quality reputation decreasing from the narrow through the wider area.

Thus, geographical origin of products can be valued by consumers either in itself or as a quality cue, that is as a feature that suggests others qualities. If it were possible to measure consumer preferences for a food before and after releasing information about the geographical origin, the difference between the two measures would provide an assessment of the direct impact of the region of origin. However, at least for sensory attributes, reality seems to be somewhat more garbled as sensory perceptions are not stable but can change depending on which non-sensory information is made available to the consumer.

Following the classification provided by Schifferstein (2001), three alternative methods to elicit sensory preferences can be identified depending on the information set available to individuals: blind taste, expectation (provision of non-sensory information only) and labelled tests (provision of non-sensory information such as a label and tasted samples).

In the three cases preferences can be measured through hedonic scores. Let be $B, E$ and $L$ the scores respectively measured in the blind, expectation and labelled tests. The differences between scores under different information regimes are denominated as follows (Schifferstein, 2001):

$$
\begin{aligned}
& L-E=\text { degree of disconfirmation, } \\
& E-B=\text { degree of incongruence, } \\
& L-B=\text { degree of response shift. }
\end{aligned}
$$


To single out the direct impact of region of origin on the valuation, a promising measure seems to be the monetary equivalent of the response shift. Such a measure would allow a disentanglement of the impact of the process characteristic on valuation as a quality cue from the direct impact of the same due to its "non-use" value. ${ }^{2}$

However, actual sensory perceptions are not independent from sensory expectations derived from extrinsic cues. When the process characteristics refer to the geographical origin of the product it has been reported that consumers indifferent between two product in the blind test showed a strong sensory preference for products produced in specific areas in the labelled test (Guerrero, 2001). One of the theories that try to explain this empirically observed pattern is the assimilation theory. According to it, disconfirmed expectations cause a state of psychological pain in consumers as prior beliefs are contradicted by experience. Therefore, consumers reduce their pain altering their sensorial perception in the direction of expectations (Anderson, 1973; Deliza \& MacFie, 1996; Schifferstein, 2001). In other words the assimilation theory predicts that in the labelled test consumers may try to be consistent with the expectation they formed when rating the non-sensory (label) information. To some extent, this refers to what happens in real life where consumers are usually exposed to nonsensory information (packaging, advertisement) before trying the product itself.

Assimilation can be measured by the ratio $(\alpha)$ of the response shift over the incongruence degree:

$\alpha=(L-B) /(E-B) \quad$ with $1 \geqslant \alpha \geqslant 0$.

When the external cues do not affect the sensory valuation $\alpha$ equals zero, whilst the irrelevance of sensory characteristics on the overall evaluation gives rise to a ratio of 1 (Lange, Rousseau, \& Issanchou, 1999). When assimilation is absent and extrinsic cues do not interfere with sensory perceptions the blind test score should be equal to the labelled test score and $\alpha$ should be equal to 0 .

This strand of literature provides interesting insights on the effects of information on sensory characteristic perception and related hedonic measures. Conversely, few works have related sensory measures with willingness to pay (Lange, Rousseau, \& Issanchou, 1998; Lange, Martin, Chabanet, Combris, \& Issanchou, 2002; Melton, Huffman, \& Shogren, 1996; Melton, Huffman, Shogren, \& Fox, 1996; Umberger \& Feuz, 2004). According to most of these works, hedonic scores mea-

\footnotetext{
${ }^{2}$ Here the term 'non-use' refers to the value of process characteristics that is not related to their direct consequences on sensory characteristics. For example, in the case of low impact production processes response shift would allow to disentangle the impact of this process characteristics on how food is valued because of better taste from the willingness to pay to preserve the environment.
}

sured through liking scales seem to be positively correlated with willingness to pay measures. However, Lange et al. (2002) acknowledge that WTP experiments are more sensitive in detecting product differences when researching the perceived value of a brand or when the product is tested in labelled conditions, while hedonic measurement under blind condition is more valuable to assess "the intrinsic value" of a product. This suggestion has not been further investigated. To the knowledge of the authors, in the case of specialty food, no study so far has tried to research whether monetary valuations account not only for use values conveyed by sensory characteristics, but also for non-use values related to the ethical, affective and normative factors and under which conditions non-use values are relevant.

The aim of this work is to analyse the impact of geographical origin on consumers' willingness to pay for a food specialty drawing on the marketing literature on the direct (affective and normative) role of region of origin and on previous research on the comparison between hedonic scoring and WTP. The research focuses on three types of spelt produced in three concentric areas: Garfagnana, Tuscany and Italy Assessed under blind, expectation and labelled test conditions, that provide consumers with a mix of information about sensory characteristic, geographical origin or a combination of the two respectively.

The experiment was devised to pursue two specific objectives: (i) to assess the impact of information about the geographical origin of the product on the level of WTP for spelt produced in different regions, and (ii) to measure the direct effect of different definition (wide vs narrow) of the region of origin on WTP taking into account the correspondent changes in sensory perceptions as well whenever assimilation is detected.

\section{Materials and methods}

Both hedonic scores and WTP were elicited within an experimental design that combined hedonic tests and experimental auctions replicated over six sessions. The treatments were geographical origin (three levels: Garfagnana, Tuscany and Italy) and information condition (blind test, expectation test and labelled test). Within each session, subjects first participated into a blind test indicating their "liking" evaluation for servings of spelt from the three areas. They were then requested to bid for samples of the same spelt they had previously tasted. Afterwards, participants were provided with labels of spelt. Liking expectations were elicited and then a second auction followed. Finally, a labelled test took place and a last auction concluded the experiment. At the end, participants were asked to fill in a self-administered questionnaire on demographics characteristics. 


\subsection{Participants}

A sample of 111 shoppers (42 males and 69 females) aged between 25 and 70 was selected through face to face screening interviews at a commercial outlet (COOP hypermarket) in the metropolitan area of Florence in Tuscany (Italy). Besides age and shopper status, a third screening variable concerned spelt consumption. Subjects were selected only if they had consumed spelt at least once in the previous year. A quota sampling by age (25-40 and 41-70) and gender was adopted in order to reproduce the characteristics of the average customer of the outlet. Out of the 111 contacted subjects that agreed to take part into the experiment 77 (30 males and 47 females) showed up at the venue located in the office building of the commercial outlet. Experimental sessions took place at 5 p.m. in six days of the week (from Thursday through to the following Wednesday excluding Sunday). Hedonic scores and WTP were elicited following the experimental protocol described below.

\subsection{Products}

Auctioned samples were packs of $500 \mathrm{~g}$ of spelt produced in the three areas. Sensory analysis was performed on servings of boiled spelt. To avoid first position distortions and possible carry over effects a William's latin square design was employed (MacFie, Bratchell, Greenho, \& Vallis, 1989).

Labels for expectation and labelled tests (Fig. 1) were printed in black and white on A4 sheets partially reproducing the same lay-out of commercial labels. Each label shows information about weight of package, culinary use of spelt and geographical origin. Furthermore a small map of Italy was added with the production area of each spelt type blackened.

\subsection{Hedonic scores and Vickrey auctions}

Hedonic scores were elicited making participants express their evaluations by ticking on a $12 \mathrm{~cm}$ linear scale anchored at the right end with "I like it very much" and at the left end with "I do not like it at all". In the case of expectation tests the previous anchoring were replaced by "I would like it very much" and "I would not like it at all".

A more complex procedure was employed to elicit WTP. Traditionally, in experimental economics one of the widely adopted mechanism is the so-called Vickrey auction (Vickrey, 1961), whereby participants submit sealed bids and the winner is the highest bidder but pays only a price corresponding to the second highest bid. This auction mechanism induces participants to bid their true reservation price (their maximum WTP) for the auctioned good. In this research a $n$th random price format was adopted. In this format the price is not determined by the second highest bid but rather by the $n$th highest bid where $n$ is a number greater than or equal to 2, but smaller than the number of participants. The number $n$ is randomly drawn at the end of the auction. Consequently, the number of winners, set to $n-1$, is only known after bids have been submitted. This feature assures a greater involvement of participant submitting very low bids (off margin bidders) who face a very low probability of winning and making real payments in standard Vickrey auctions (Shogren, Margolis, Koo, \& List, 2001).

\subsection{Experimental protocol}

The adopted experimental protocol is similar to the one proposed by Lange et al. (2002) to evaluate the impact of label information on sensory liking and WTP for Champagne bottles. The main difference between the two is that Lange et al. employed classic second price
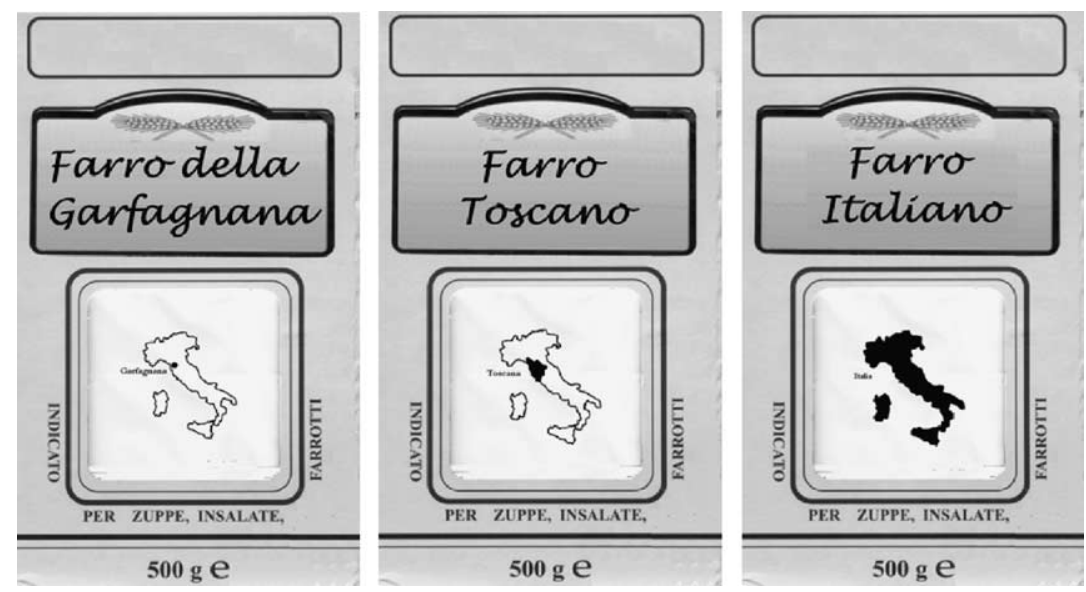

Fig. 1. Labels for expectation and labelled tests. 
Table 1

Experimental protocol

\begin{tabular}{ll}
\hline Steps & Description \\
\hline Training & Hedonic evaluation of chocolate bars (only expectation test) \\
& $n$th random price auction of chocolate bars \\
Blind test & Tasting and hedonic scores elicitation (one type at any one time) \\
& Simultaneous $n$th random price auction of spelt packs \\
Expectation test & Evaluation of labels and scores elicitation (one type at any one time) \\
& Simultaneous $n$th random price auction of spelt packs \\
Labelled test & Tasting of labelled spelt types and hedonic scores elicitation (one type at any one time) \\
& Simultaneous $n$th random price auction of spelt packs \\
Purchases & Drawing of random numbers. Proclamation of winners and carrying out of real purchases \\
Final questionnaire & Self-administered questionnaire filling
\end{tabular}

Vickrey auctions while in this work the random $n$th price format was used.

At the beginning of each session participants were given printed instructions stating that they were to participate in hedonic evaluations and auctions for three types of spelt under three information condition: blind, expectation and labelled testing. They then signed a consent form and were endowed with $€ 35$ as an incentive. The experimenter strictly read the printed instructions in order to minimize session effects. The experiment consisted of two stages broken down into a series of steps (Table 1). The first stage (training) was performed with chocolate bars and aimed at familiarizing participants with scales used to elicit hedonic scores and auction procedures. Then the actual spelt stage began.

It was explained to the participants that they were to successively taste three portions of spelt under blind condition and would be requested to indicate their liking using the linear scale previously described. Then subjects were reminded of the rules of $n$th random price auction already employed in the training stage. They were also told that they could choose the maximum number of spelt packs they would have to buy in case they won more than one auction. This was devised to deal with possible income effects caused by multiple winnings (Melton, Huffman, Shogren, \& Fox, 1996). Indeed, without this device, low income people who bids higher in the earlier auctions might bid lower than their reservation price in the subsequent auctions, fearing that a higher number of winnings would translate in a relatively large out of pocket expense. An auction was then performed simultaneously for the three types of spelt (indicated as 1, 2 and 3) corresponding to the samples tasted in the blind test. Bids were submitted by ticking the price on three graduated scales ranging from 0 to 5 euro with divisions corresponding to 10 eurocents. Noticeably, before bidding each participant was provided with the hedonic scores he had assigned to spelt types in the blind test.

For the other two condition of information (expectation test and labelled test) the same steps described for the blind test condition were performed. Overall, nine auctions ( 3 conditions of information $\times 3$ geographical origins) were carried out for spelt packs. At the end of each experimental session the numbers that determined market price and number of winners for each auction were drawn. Thus, if the drawn number had been 5 the market price would have been set to the fifth highest bid and the four participants with the highest bids would have won the auction. Winners were subsequently proclaimed and real monetary transactions (i.e. purchases) took place.

\subsection{Data analysis}

Means across treatment cells (condition of information $\times$ region of origin) were first calculated. Since a within subjects experimental design was adopted, analysis were performed also on centered data. Thus, the subject mean value for WTP and hedonic scores were calculated across all nine treatment cells and subtracted from actual figures. ${ }^{3}$ Subsequently, an analysis of variance with repeated measures was carried out to investigate the role of factors affecting WTP.

In order to explore the role of the direct impact of geographical origin on value, the means of the monetary equivalent of the response shift (difference between WTP under labelled and blind test conditions) were computed for the three regions of origin. Both parametric paired difference $t$-tests and non-parametric Wilcoxon signed rank test (Cramer, 1994) were performed.

The presence of assimilation was assessed through the ratio $\alpha$ (see Section 2 above) obtained by regressing the response shift on the incongruence degree on both hedonic scores and WTP. Correlation between centered hedonic scores and WTP were calculated as well across conditions of information.

Finally, because of the panel nature of data, the marginal impact of regions of origin on WTP was assessed through a random effect panel model.

\footnotetext{
${ }^{3}$ The authors thank an anonymous referee for suggesting this data transformation.
} 


\section{Results}

\subsection{Impact of geographical origin on WTP}

Mean WTP and hedonic scores observed in the different treatment combinations are reported in Table 2. Evaluations of spelt seem to change when information about the origin is released. Noticeably, the average sensory and monetary valuation of Garfagnana spelt expressed after the expectation test is higher than the valuation obtained under blind test conditions, while the opposite pattern is observed in the case of Italian spelt.

Factors affecting WTP are analysed through an analysis of variance model with repeated measures to take into account the subject effect. Besides the two treatment factors a session factor is added to deal with the variability caused by unanticipated factors such as those related to the composition of the group. Results show significant effects for both geographical origin and the interaction origin $\times$ condition of information (Table 3 ). Interaction between session effects and treatment factors is not significant.

Interaction between origin and condition of information can be better appreciated through the graphical representations set out in Figs. 2 and 3. To be consistent with the repeated measure factor analysis centered data is used. A crossover effect emerges as valuations of the three spelt types follow different patterns when shifting across conditions of information. Impact of information on geographical origin is stronger for both Italy and Garfagnana, though in an opposite way. These origins can be ideally placed at the two ends of an index related to the degree of typicality being the largest and the narrowest areas of production under investigation.

Both monetary and hedonic valuations under condition of full information (labelled test) show minor changes with respect to those stated after the expectation test. Participants adjusted their valuations for Italian spelt upwards while a downward correction was observed for Garfagnana spelt. Conversely, WTP for Tuscan spelt does not change significantly across condi-
Table 3

Analysis of variance (within effects), WTP

\begin{tabular}{lcrl}
\hline Within effects & $\begin{array}{l}\text { Degrees of } \\
\text { freedom }\end{array}$ & $F$ & $p$-Level \\
\hline Session & 5 & 3.67 & 0.01 \\
Condition of information & 2 & 2.54 & 0.08 \\
Origin & 2 & 16.03 & 0.00 \\
Session $\times$ condition of information & 10 & 0.53 & 0.86 \\
Session $\times$ origin & 10 & 1.17 & 0.32 \\
Condition of information $\times$ origin & 4 & 11.49 & 0.00 \\
Session $\times$ cond. inf. $\times$ origin & 20 & 0.33 & 1.00 \\
\hline
\end{tabular}

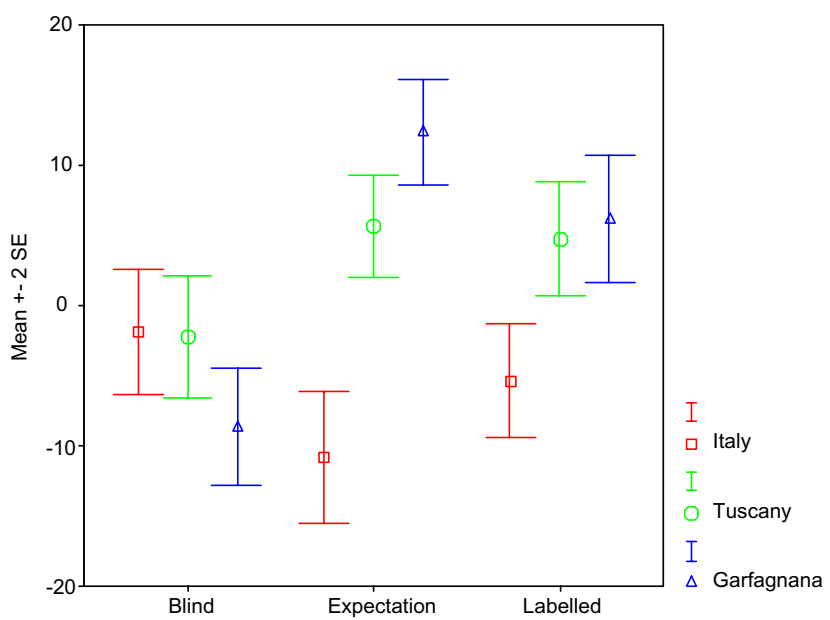

Fig. 2. Interaction effects (geographical origin $\times$ condition of information) on hedonic scores (deviations from subject means).

tions of information as it appears geographical origin had not had a relevant impact on valuations.

Overall, information about geographical origin appears to have a significant impact on the evaluation of spelt types, thereby confirming the findings of other works (Van der Lans et al., 2001; Von Alvensleben \& Schrader, 1998). However, it is not clear whether geographical origin is simply a cue for other characteristics or it is valuable in itself. To address this point both WTP and hedonic scores will be analysed employing a monetary equivalent of the response shift following the suggestions set out in Section 2.

Table 2

Mean WTP $(€)$ : spelt type and information condition

\begin{tabular}{|c|c|c|c|c|c|c|}
\hline \multirow[t]{2}{*}{ Region of origin } & \multicolumn{3}{|l|}{ WTP } & \multicolumn{3}{|c|}{ Hedonic scores } \\
\hline & Blind & Expectation & Labelled & Blind & Expectation & Labelled \\
\hline \multirow[t]{2}{*}{ Italy } & 1.37 & 1.22 & 1.29 & 50.16 & 41.22 & 46.67 \\
\hline & $(0.71)$ & $(0.64)$ & $(0.66)$ & $(23.64)$ & $(24.72)$ & $(22.77)$ \\
\hline \multirow[t]{2}{*}{ Tuscany } & 1.41 & 1.43 & 1.45 & 49.78 & 57.66 & 56.77 \\
\hline & $(0.74)$ & $(0.69)$ & $(0.71)$ & $(23.62)$ & $(20.98)$ & $(24.19)$ \\
\hline \multirow[t]{2}{*}{ Garfagnana } & 1.29 & 1.59 & 1.52 & 43.37 & 64.40 & 58.20 \\
\hline & $(0.70)$ & $(0.73)$ & $(0.74)$ & (21.89) & (22.88) & $(23.51)$ \\
\hline
\end{tabular}

Standard deviations in brackets. 


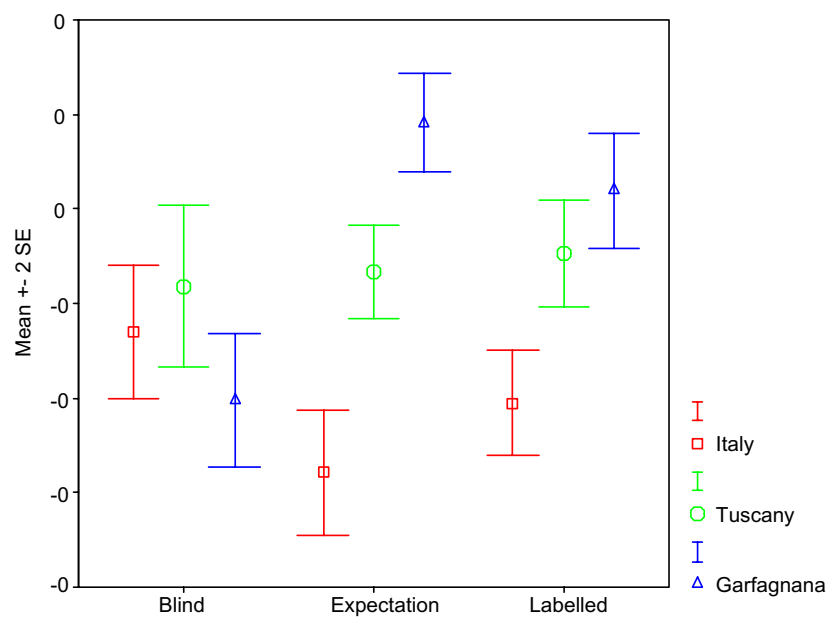

Fig. 3. Interaction effects (geographical origin $\times$ condition of information) on WTP (deviations from subject means).

\subsection{Direct impact of geographical origin}

The direct effect of geographical origin can be measured taking the difference between WTP under full information (labelled test) and WTP under blind test condition for a given origin and comparing this difference with the corresponding one observed for a baseline origin. As mentioned above (see Section 2), the labelledblind difference is the monetary equivalent of the response shift measure for hedonic scores.

Taking as reference the largest area of production (Italy), direct impacts for Garfagnana $\left(D_{\mathrm{G}}\right)$ and Tuscany $\left(D_{\mathrm{T}}\right)$ origins are given by the following expressions:

$$
\begin{aligned}
D_{\mathrm{G}}= & \left(\mathrm{WTP}_{\mathrm{G}_{\text {labelled }}}-\mathrm{WTP}_{\mathrm{G} \text { blind }}\right) \\
& -\left(\mathrm{WTP}_{\text {Ilabelled }}-\mathrm{WTP}_{\mathrm{I} \text { blind }}\right) \\
D_{\mathrm{T}}= & \left(\mathrm{WTP}_{\mathrm{T} \text { labelled }}-\mathrm{WTP}_{\mathrm{T} \text { blind }}\right) \\
& -\left(\mathrm{WTP}_{\text {Ilabelled }}-\mathrm{WTP}_{\mathrm{I} \text { blind }}\right)
\end{aligned}
$$

The presence of a direct impact implies a significant difference between the monetary response shifts. When comparing Garfagnana to Italy, both parametric and non-parametric ${ }^{4}$ tests for differences of means in paired samples are significant at the 0.01 level (Table 4). The impact of Tuscan origin is less clear because the difference is significant only at the 0.10 level. In summary, comparisons of mean response shifts does not allow the rejection of the presence of a direct impact of geographical origin, at least for the Garfagnana area.

However, such a conclusion would be invalidated if assimilation is present. Indeed, monetary response shifts analysed in Table 4 can measure the direct value of geo-

\footnotetext{
${ }^{4}$ A Wilcoxon signed rank test was employed.
}

graphical origin only if sensory perceptions do not change under different information conditions.

The presence of assimilation of perceived towards expected liking can be checked through the ratio $\alpha$ obtained by regressing the response shift on the incongruence degree. The measure based on hedonic scores (Fig. 4) gives a value of $\alpha=0.71$ with a standard error of 0.05 suggesting that participants weight more the expectation based on the area of origin than the actual trial information.

A corresponding measure could be implemented to investigate differences in WTP (Fig. 5). In this case the ratio is higher $(\alpha=0.80$; standard error $=0.04)$ but not significantly different from the previous one at the 0.05 level, giving no clear indication about a higher impact of information regarding geographical origin in the case of monetary valuations.

It is worth noticing that in the hedonic score case the share of variance of response shift explained by the degree of incongruence was smaller $\left(R^{2}=0.50\right)$ than in the WTP case. Subjects showed a more homogeneous behaviour for auction bids than for hedonic scores. The same pattern was also reported by Lange et al. (2002).

The assimilation found with respect to hedonic scores causes a bias in the estimation of the direct effect of geographical origin. A share of the observed change of WTP between labelled and blind test conditions is attributable to a parallel shift of hedonic perceptions whereby participants adapt actual perceptions to expected ones. Indeed, hedonic scores and WTP seems to be uniformly correlated across information conditions once data have been centered to take the effect of subjects into account (Table 5). A comparison with the value measured by Melton, Huffman, and Shogren (1996) in a similar experiment on monetary and sensorial evaluation of pork chops is possible only for the labelled condition and using raw rather than centered data. The obtained value is 0.25 , far lower than the one reported by Melton et al. However, in Melton's experiment, pork chops differed only on search characteristics (such as marbling, colour, size) and this feature might explain the higher correlation between WTP and hedonic scores found in his study.

To investigate to what extent observed monetary response shifts are explained by the analogous sensory measure rather than by the direct effect of geographical origin on WTP, differences in WTP (labelled-blind) were regressed on differences in hedonic scores. In order to account for the panel nature of data (as more evaluations were performed by the same subjects on different types of spelt under different conditions of information) a random effects panel model was adopted. The use of a random rather than a fixed effect model is preferable if the sampled individuals are believed to be drawn from a larger population. Moreover, subject specific variables 
Table 4

Mean WTP differences: labelled test vs blind test condition of information (response shift)

\begin{tabular}{|c|c|c|c|c|c|}
\hline Geographical origin & $\begin{array}{l}\Delta \text { WTP labelled } \\
\text { vs blind }(€)\end{array}$ & $\begin{array}{l}t \text {-Test for differences between } \\
\text { means for dependent samples }\end{array}$ & $p$ & $\begin{array}{l}Z \text { Wilcoxon matched pair test } \\
\text { for differences between means }\end{array}$ & $p$ \\
\hline Tuscany vs Italy & $\begin{array}{r}0.04 \\
-0.08\end{array}$ & 1.70 & 0.09 & 1.77 & 0.08 \\
\hline Garfagnana vs Italy & $\begin{array}{r}0.22 \\
-0.08\end{array}$ & 4.62 & 0.00 & 4.34 & 0.00 \\
\hline
\end{tabular}

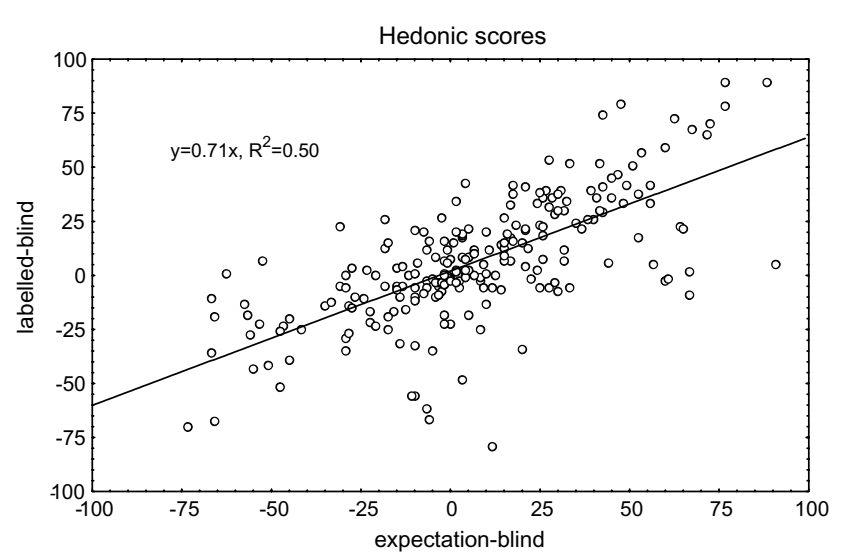

Fig. 4. Assimilation effects on hedonic scores.

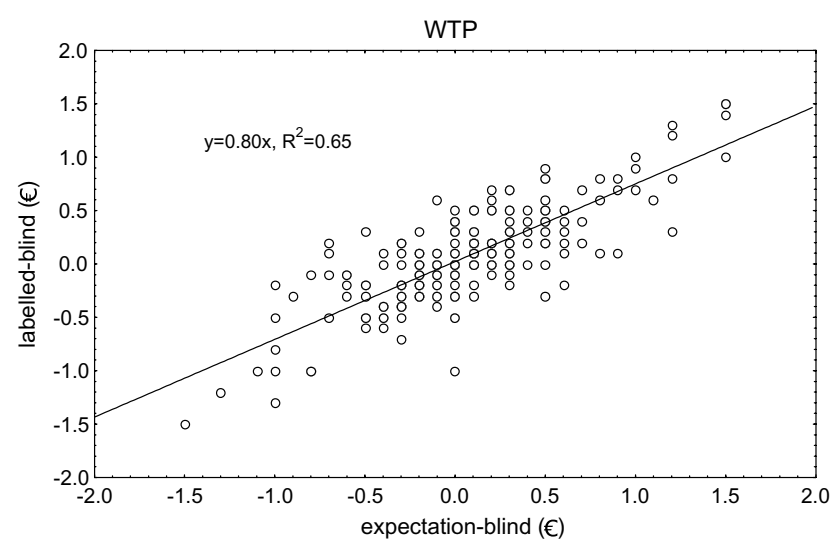

Fig. 5. Assimilation effects on WTP.

such as age or sex can be added to the regressors only within a random effect specification. ${ }^{5}$ The estimated linear model is given by

$\Delta \mathrm{WTP}_{i t}=\gamma_{i}+\boldsymbol{\beta} \mathbf{x}_{i t}+u_{i}+\varepsilon_{i t}$

where $\Delta \mathrm{WTP}_{i t}$ is the difference between WTP under full information (labelled test) and WTP under blind test condition for the $i$ th participant and the $t$ th spelt type,

\footnotetext{
${ }^{5}$ Orthogonality between random effects and regressors which is required in order to get consistent estimates within a random effect models was tested and could not be rejected at the 0.05 level according to the Hausman test. See Greene (2000) for a discussion of random effect linear model with panel data.
}

Table 5

Correlation coefficient between centered hedonic scores and WTP

\begin{tabular}{lll}
\hline Test conditions & \multicolumn{2}{l}{ Correlation coefficient } \\
\cline { 2 - 3 } & $\rho$ & Std. Err. \\
\hline Blind test & 0.564 & 0.055 \\
Expectation test & 0.629 & 0.051 \\
Labelled test & 0.564 & 0.055 \\
Overall & 0.587 & 0.031 \\
\hline
\end{tabular}

To obtain centered variables the subject means across all treatment cells were subtracted from actual data.

$\gamma$ is a constant, $\mathbf{x}_{i t}$ is a vector of independent variables (including differences between labelled and blind test hedonic scores and dummy variables on geographical origin, auction session and demographic characteristics of participants), $\beta$ is a conformable vector of coefficients, $u_{i}$ is a participant specific error term and $\varepsilon_{i t}$ is the overall error term.

The use of dummy variables for Garfagnana and Tuscany in Eq. (4) is devised to estimate the marginal impact of these areas of origin with respect to Italy (whose dummy was dropped to avoid the dummy trap problem) on the monetary response shift thus giving a test similar to the one illustrated by relations (2) and (3).

Regression coefficients are statistically significant only for the Garfagnana origin dummy and for the difference in hedonic scores (Table 6). The lack of significance of demographic variables is not surprising as these variables are likely to show a stronger impact on levels of WTP rather than on differences. Secondly, their influence could have been already captured, at least in part, by differences between hedonic scores.

A significant coefficient for sensory response shift was expected, having been found by other studies. For example, Umberger and Feuz (2004) in a work on beef steak observed a significant effect of relative hedonic scores on relative WTPs.

More interestingly, a significant (at the 0.05 level) coefficient for Garfagnana origin means that once changes in sensory perceptions have been taken into account there is still a marginal impact of this area of origin on WTP. Considering that the dummy variable coefficient represents an intercept shift with respect to the Italian origin, a greater and significant impact of the narrowest area of production support the hypothesis that a more precise definition of the area brings about a 
Table 6

Impact of $\Delta$ hedonic scores, geographical origin and demographics on $\Delta$ WTP: random effect model

\begin{tabular}{|c|c|c|c|c|}
\hline Variables & Coefficient & Std. Err. & $t$-Ratio & $p$-Value \\
\hline Constant & -0.01 & 0.14 & -0.04 & 0.97 \\
\hline$\Delta$ Liking (labelled-blind) & 0.01 & 0.00 & 10.54 & 0.00 \\
\hline Garfagnana origin & 0.13 & 0.05 & 2.52 & 0.01 \\
\hline Tuscany origin & 0.02 & 0.05 & 0.33 & 0.74 \\
\hline Auction 1 & 0.05 & 0.11 & 0.48 & 0.63 \\
\hline Auction 2 & 0.09 & 0.10 & 0.91 & 0.36 \\
\hline Auction 3 & -0.06 & 0.10 & -0.58 & 0.56 \\
\hline Auction 4 & 0.03 & 0.10 & 0.29 & 0.77 \\
\hline Auction 5 & 0.10 & 0.11 & 0.93 & 0.35 \\
\hline Gender $(\mathrm{M}=1 ; \mathrm{F}=0)$ & -0.04 & 0.06 & -0.63 & 0.53 \\
\hline Age $(25-40=1 ; 41-70=0)$ & -0.02 & 0.07 & -0.29 & 0.77 \\
\hline Education $($ secondary or high school $=1 ;$ otherwise $=0$ ) & -0.09 & 0.06 & -1.37 & 0.17 \\
\hline Self-reported financial status (very well off or well off $=1$; difficult or modest $=0$ ) & 0.00 & 0.05 & -0.01 & 0.99 \\
\hline$R^{2}$ & 0.34 & & & \\
\hline Number of observations & 231 & & & \\
\hline
\end{tabular}

higher degree of typicality, thereby enhancing the nonuse value of geographical origin.

\section{Discussion and conclusion}

The results of this research highlight a complex pattern of relationships among monetary valuations, hedonic scores and the definition of the area of origin of typical food products. In the case of spelt in Italy, both region of origin and the interaction of this factor with information condition (blind, expectation and labelled tests) significantly affect hedonic scores and WTP for spelt packs in experimental auctions.

The adopted design allows the investigation of the relationship between hedonic scores and WTP more closely, further developing suggestions advanced by Lange et al. (2002) about the different nature of the two measures. Indeed, results suggest that in the presence of symbolic and affective components of the value, hedonic scores and WTP measures are not directly comparable, the latter including more value components than the former.

In addition, the design disentangles the affective or symbolic value of the geographical origin from the overall evaluation of the product. Thus, at least for foodstuffs, this paper suggests a procedure to operationalise the monetary assessment of a source of value well investigated by the marketing literature (Verlegh \& Steenkamp, 1999). This knowledge may be important when designing the marketing mix for regional specialties.

It would be interesting to further investigate how possible modifications of the adopted design would impact on results. For example, Lange et al. (1999) suggest that consumers might well change their expectation if exposed to multiple disconfirmations. Furthermore, the order of presentation of the blind, expectation and labelled stimuli might affect the results as ratings under labelled test conditions might be influenced by mental constructions elaborated when assessing the label information as suggested by Scifferstein (1997). Future research should investigate how the order of presentation and the reiteration of treatments impact on results. Generally, it seems important to further study the process and timing of expectation formation induced by origin labels under different experimental settings trying, as far as possible, to parallel real world features of the purchase process.

From a marketing point of view, it seems important to develop protocols that investigate the impact of more complex product information. In particular, price information is likely to affect subjects' WTP responses. Lange et al. (2002) induced a lower bound to the bid distribution in their experiment by announcing to participants the existence of a reservation price. These aspects of the experimental protocol should be further explored.

As far as the definition of the area of origin is concerned, the outcomes of the experiment show that the narrower defined the area, the higher the expected value of the food. Noticeably, an assimilation effect is observed whereby consumers weigh more the expectations induced by the origin than the actual trial information. Indeed, origin from Garfagnana, a small mountainous area of Tuscany, evokes strong expectations of quality that are capable to positively influence both sensory and monetary valuations in expectation and labelled tests. The wider regional definition (Tuscany) still shows a positive impact on consumer evaluation as compared to the Italian origin. This suggests that even for products such as Spelt, a concentric structure of the region of origin denomination could be potentially implemented.

Moreover, in the case of spelt, origin from small areas such as Garfagnana seems also to have a significant direct impact on food evaluation confirming results from marketing studies. Whether this effect is entirely attributable to non-use values of the region of origin, such 
as the appreciation for culinary heritage and tradition or to other factors is still an open question. Alternative candidate explanations such as the subjects' uncertainty of the exact origin when wider definitions of the area are adopted $^{6}$ do not seem convincing but should be further explored. The rationale behind this explanation is that if consumer are risk averse, the presence of increasing uncertainty should lower their WTP. However, in real life, if concentric areas are uniformly ranked by all consumers, the two regions of origin - say Garfagnana and Tuscany - are considered by consumers as complements rather than one the subset of the other. In fact, consumers know that there would be no advantage for the producers to label the good originating from the narrower region, i.e. Garfagnana, as the ones produced in the wider region, i.e. Tuscany, because discriminating between the two regions would be more profitable.

In conclusion, further research is needed to address methodological issues and explore other possible explanations of the direct impact of origin on values. Also, it would be interesting to study other types of food specialities for which we might hypothesise a different weight for sensory characteristics and the area of origin in the preference formation process.

\section{Acknowledgements}

This research was funded by the Italian Institute of research on the Mountain (INRM) and the Tuscan Agency for Development and Innovation in Agriculture (ARSIA). Research support from UNICOOP Firenze is gratefully acknowledged. The authors benefited from insightful comments by Jason Shogren.

\section{References}

Anderson, R. E. (1973). Consumer dissatisfaction: the effect of disconfirmed expectancy on perceived product performance. Journal of Marketing Research, 10(2), 38-44.

Bell, D., \& Valentine, J. (1997). Consuming geographies. London: Routledge.

Bessière, J. (1998). Local development and heritage: traditional food and cuisine as tourist attractions in rural areas. Sociologia Ruralis, 38(1), 21-34.

Cramer, D. (1994). Introducing statistics for social research. London: Routledge.

Deliza, R., \& MacFie, H. J. H. (1996). The generation of sensory expectation by external cues and its effect on sensory perception and hedonic ratings: a review. Journal of Sensory Studies, 11, 103-128.

Friedman, M. (1996). A positive approach to organized consumer action: the "Buycott" as an alternative to the boycott. Journal of Consumer Policy, 19, 439-451.

Greene, W. (2000). Econometric analysis. Upper Saddle River, NJ: Prentice-Hall.

\footnotetext{
${ }^{6}$ The authors are indebted to one of the anonymous referee for this suggestion.
}

Grunert, K. G., Larsen, H. L., Madsen, T. K., \& Baadsgaard, A. (1996). Market orientation in food and agriculture. Norwell, MA: Kluwer Academic Publishers.

Guerrero, L. (2001). Marketing PDO and PGI. In L. Frewer, E. Risvik, \& H. Schifferstein (Eds.), Food, people and society (pp. 281-298). Berlin: Springer.

Lange, C., Martin, C., Chabanet, C., Combris, P., \& Issanchou, S. (2002). Impact of the information provided to consumers on their willingness to pay for champagnes: comparison with hedonic scores. Food Quality and Preference, 13(7-8), 597-608.

Lange, C., Rousseau, F., \& Issanchou, S. (1998). Expectation, liking and purchase behaviour under economical constraint. Food Quality and Preference, 10(1), 31-39.

Lange, C., Rousseau, F., \& Issanchou, S. (1999). Expectation, liking and purchase behaviour under economical constraint. Food Quality and Preference, 10, 31-39.

MacFie, H. J., Bratchell, N., Greenho, K., \& Vallis, L. V. (1989). Designs to balance the effect of order of presentation and firstorder carry-over effects in hall tests. Journal of Sensory Studies, 4 , $129-148$.

Melton, B. E., Huffman, W. E., \& Shogren, J. F. (1996). Economic values of pork attributes: hedonic price analysis of experimental auction data. Review of Agricultural Economics, 18, 613627.

Melton, B. E., Huffman, W. E., Shogren, J. F., \& Fox, J. A. (1996). Consumer preferences for fresh food items with multiple quality attributes: evidence from an experimental auction of pork chops. American Journal of Agricultural Economics, 78, 916-923.

Moran, W. (1993). Rural spaces as intellectual property. Political Geography, 12, 263-277.

Ray, C. (1998). Culture, intellectual property and territorial rural development. Sociologia Ruralis, 38(1), 1-20.

Scifferstein, H. (1997). Perceptual and imaginary mixture in chemosensation. Journal of Experimental Psychology Human Perception and Performance, 23(1), 278-288.

Schifferstein, H. (2001). Effects of product beliefs on product perception and liking. In L. Frewer, E. Risvik, \& H. Schifferstein (Eds.), Food, people and society (pp. 73-96). Berlin: Springer.

Shogren, J. F., Margolis, M., Koo, C., \& List, J. A. (2001). A random $n$ th-price auction. Journal of Economic Behaviour and Organisation, 46, 409-421.

Steenkamp, J. B. (1990). Conceptual model of the quality perception process. Journal of Business Research, 21(4), 309-333.

Umberger, W. J., \& Feuz, D. M. (2004). The usefulness of experimental auctions in determining consumers' willingness-topay for quality-differentiated products. Review of Agricultural Economics, 26(2), 170-185.

Van der Lans, I. A., Van Ittersum, K., De Cicco, A., \& Loseby, M. (2001). The role of the region of origin and EU certificates of origin in consumer evaluation of food products. European Review of Agricultural Economics, 28(4), 451-477.

Van Ittersum, K., Candel, M., \& Meulemberg, M. (2003). The influence of the image of a product's region of origin on product evaluation. Journal of Business Research, 56, 215-226.

Verlegh, P. W. J., \& Van Ittersum, K. (2001). The origin of spices: the impact of geographic product origin on consumer decision making. In L. Frewer, E. Risvik, \& H. Schifferstein (Eds.), Food, people and society (pp. 267-280). Berlin: Springer.

Verlegh, P. W. J., \& Steenkamp, J. B. (1999). A review and metaanalysis of country of origin research. Journal of Economic Psychology, 20, 521-546.

Von Alvensleben, R., \& Schrader, S. K. (1998). Consumer attitudes towards regional food products. A case study for Northern Germany. AIR CAT workshop consumer attitudes toward typical foods, Dijon, 22-10-1998.

Vickrey, W. (1961). Counterspeculation, auctions and competitive sealed tenders. Journal of Finance, 16, 8-37. 